\title{
On the alleged nonlocal and topological nature of the molecular Aharonov-Bohm effect
}

\author{
Erik Sjöqvist* \\ Department of Quantum Chemistry, Uppsala University, \\ Box 518, Se-751 20 Uppsala, Sweden
}

October 31, 2018

\begin{abstract}
The nonlocal and topological nature of the molecular AharonovBohm (MAB) effect is examined for real electronic Hamiltonians. A notion of preferred gauge for MAB is suggested. The MAB effect in the linear + quadratic $E \otimes \varepsilon$ Jahn-Teller system is shown to be essentially analogues to an anisotropic Aharonov-Casher effect for an electrically neutral spin $-\frac{1}{2}$ particle encircling a certain configuration of lines of charge.
\end{abstract}

Key words: Molecular Aharonov-Bohm effect, locality, topology, Berry's phase

*Electronic address: erik.sjoqvist@kvac.uu.se 


\section{Introduction}

The molecular Aharonov-Bohm (MAB) effect, first hinted at by LonguetHiggins and coworkers 1, 2, 3, 4, is one of the paradigmatic examples on early anticipations of Berry's discovery [5] of geometric phase factors accompanying cyclic adiabatic changes. The importance of MAB ranges from testable shifts of the vibronic energy spectrum [6, 7, effects on cross-sections in molecular reactions [8, 9, 10, and effects on reduction factors [11], to subtle symmetry assignments of vibronic states in Jahn-Teller systems [12, 13, 14, 15] and search for conical intersections [4, 16, 17, 18, 19, 20. However, it is probably fair to say that interest in the MAB effect itself arose first after it was realized [21, 22] its mathematical analogy with the standard Aharonov-Bohm (AB) effect [23]. This mathematical analogy is the independence of details of the shape of the closed path in a multiply connected region of nuclear configuration space.

In a recent work [24, the present author has considered the physical nature of this analogue in the case of the $E \otimes \varepsilon$ Jahn-Teller system. The question addressed there was: does MAB share all the remarkable properties of AB? The main outcome of this analysis was that although MAB obeys the above mentioned independence of details of the closed path in nuclear configuration space, it does not share the remaining nonlocal and topological properties of $\mathrm{AB}$. The purpose of the present paper is to develop the local and nontopological nature of MAB further. In particular, we wish to extend 24] to any molecular system with real electronic Hamiltonians. We also wish to propose a notion of preferred gauge for MAB in this case, in terms of a so-called gauge invariant reference section [25, 26] that appears naturally in the theory of the open path Berry phase [27, 28].

The starting point for the argumentation in [24] is a set of criteria for a nonlocal and topological phase effect, first explicitly introduced by Peshkin and Lipkin [29]. These are:

- A phase effect is nonlocal if:

(N1) the system experiences no physical field,

(N2) no exchange of physical quantity takes place along the system's path.

- A phase effect is topological if: 
(T1) it requires the system to be confined to a multiply connected region,

(T2) any assignment of phase shift along the system's path is necessarily gauge dependent and thus neither objective nor experimentally testable.

One can argue that AB fulfills all these criteria for a nonlocal and topological phase effect. On the other hand, in the case of MAB, where the physical system consists of the nuclear configuration and a set electronic variables, the situation is very different. First, MAB is local in that it obeys neither (N1) nor (N2): the nuclei experiences the Coulomb field from the electrons, and there must be a local exchange of force to create the change in the electronic state necessary for the appearance of points in nuclear configuration space, across which the electronic open path Berry phase factor changes sign discontinuously [30, 31, 32, 33. Secondly, MAB is nontopological in that (T2) fails since there is an objective and experimentally testable open path electronic Berry phase that causes the effect on the nuclear motion. Only (T1) is fulfilled for MAB: the nuclei must be confined to a multiply connected region for the effect to occur. We wish to discuss the above criteria in relation to MAB systems of general kind.

In the next section, we extend the argumentation of [24] to the case of arbitrary molecular systems with real electronic Hamiltonians. A notion of preferred gauge for MAB in this case is suggested in section III and applied in detail to the $E \otimes \varepsilon$ Jahn-Teller system. The analogue between the MAB effect in the $E \otimes \varepsilon$ Jahn-Teller system and the Aharonov-Casher effect 34, as pointed out in 24, is further developed in section IV, so as to treat linear and quadratic coupling simultaneously. The paper ends with the conclusions.

\section{Locality and topology}

The argumentation in 24] that MAB is essentially a local and nontopological effect was put forward in the special case of the $E \otimes \varepsilon$ Jahn-Teller system. This raises the question whether the main conclusions arrived at in [24] also apply to other molecular systems that may be described accurately by real electronic Hamiltonians. In this section we address this issue and argue that $\mathrm{MAB}$ also in the general case is local and nontopological. 
For sake of clarity, we focus on the motion in some pseudorotational (internal) nuclear coordinate $\theta$, as described by the vibronic Hamiltonian

$$
H=\frac{1}{2} p_{\theta}^{2}+H_{e}(\theta)
$$

where $p_{\theta}$ is the canonical momentum corresponding to $\theta$ and $H_{e}(\theta)$ is the electronic Hamiltonian assumed to be real, traceless, and fulfilling $H_{e}(\theta+$ $2 \pi)=H_{e}(\theta)$. Let $\{|n\rangle\}_{n=1}^{N}$ be a fixed orthonormal basis of the electronic Hilbert space of finite dimension $N$. Furthermore, let $\{R(\theta) \mid \theta \in[0,2 \pi)$ be a one-parameter set of members of the rotation group $\mathrm{SO}(\mathrm{N})$ in $\mathbf{R}^{N}$, in terms of which the orthonormal instantaneous eigenvectors of $H_{e}(\theta)$ read $\{|n(\theta)\rangle=R(\theta)|n\rangle\}_{n=1}^{N}$. Then, we may write $H_{e}(\theta)=R(\theta) E(\theta) R^{\mathrm{T}}(\theta)$ with $\mathrm{T}$ being transpose and $E(\theta)=\operatorname{diag}\left[E_{1}(\theta), \ldots, E_{N}(\theta)\right]$, where $E_{1}(\theta), \ldots, E_{N}(\theta)$ are the electronic eigenenergies corresponding to $|1(\theta)\rangle, \ldots,|N(\theta)\rangle$.

The Born-Oppenheimer regime is attained when the electronic eigenenergies $\left\{E_{n}(\theta)\right\}_{n=1}^{N}$ are well separated so that the nuclear motion takes place on a single electronic potential energy surface, let us say $E_{n}(\theta)$. This may be described by the effective nuclear Hamiltonian

$$
H_{n}=\langle n(\theta)|H| n(\theta)\rangle=\frac{1}{2} p_{\theta}^{2}+E_{n}(\theta),
$$

where we have used that $\left\langle n\left|R^{\mathrm{T}}(\theta) p_{\theta} R(\theta)\right| n\right\rangle=0$. The issue of MAB arises when considering the single-valuedness of the molecular state vector $|\Psi(\theta)\rangle$, which in the Born-Oppenheimer regime is a product of the instantaneous electronic energy eigenvector $|n(\theta)\rangle$ and a nuclear factor $\chi(\theta)$, i.e., $|\Psi(\theta)\rangle=$ $\chi(\theta)|n(\theta)\rangle$. Here, we use the position representation of the nuclear motion in the pseudorotational angle $\theta$. The single-valuedness of $|\Psi(\theta)\rangle$ requires that any multi-valuedness of the electronic part must be compensated for by the nuclear part. Thus, if $|n(\theta+2 \pi)\rangle=-|n(\theta)\rangle, \chi(\theta)$ must fulfill the boundary condition $\chi(\theta+2 \pi)=-\chi(\theta)$. A nontrivial MAB effect arises if and only if there is no phase transformation of the form $|n(\theta)\rangle \rightarrow|\widetilde{n}(\theta)\rangle=e^{i \xi(\theta)}|n(\theta)\rangle$ so that the effective vector potential

$$
A_{n}(\theta) \equiv i\left\langle\widetilde{n}(\theta)\left|\partial_{\theta}\right| \widetilde{n}(\theta)\right\rangle=-\partial_{\theta} \xi(\theta)
$$

vanishes everywhere and at the same time retaining single-valuedness for $\chi(\theta)$ under the requirement that $|\Psi(\theta)\rangle$ is single-valued. 
Under the condition that there is a nontrivial MAB effect, let us ask: what is its physical nature in relation to the standard AB effect? Let us first address the issue of locality. As already indicated, the standard AB effect, which may occur when a charged particle encircles a line of magnetic flux, is nonlocal as it fulfills the criteria (N1) and (N2): the effect arises although the particle experiences no physical field and no exchange of physical quantity takes place along the particle's path. Could the same be said about the MAB effect? One way to address this question is to note that since the electronic Born-Oppenheimer states are eigenstates of $H_{e}(\theta)$, it may be tempting to replace the electronic motion by the appropriate eigenvalue of $H_{e}(\theta)$ in the Born-Oppenheimer regime so that the electronic variables can be ignored, creating an illusion that the nontrivial effect of the MAB vector potential on the nuclear motion is nonlocal and topological in the sense of the standard $\mathrm{AB}$ effect. However, this argument fails essentially because the electronic variables are dynamical and do not commute among themselves. Thus, there always exist a subset of these variables, namely those that are off-diagonal in the instantaneous electronic eigenbasis $\{|n(\theta)\rangle\}_{n=1}^{N}$, whose expectation values vanish in the Born-Oppenheimer limit, but whose fluctuations do not. These fluctuations are due to the local interaction between the nuclear variables and the electronic degrees of freedom. Thus, both (N1) and (N2) fail for MAB.

Next, we address the issue of topology. The standard AB effect is topological in that (T1) it may occur although the region of magnetic field is inaccessible to the encircling charged particle and in that (T2) the charged particle only feels the gauge dependent vector potential along the path. In the case of MAB, recall that two types of degrees of freedom are involved: those of the electrons and those associated with the nuclear configuration. Now, any assignment of nuclear phase for open paths in nuclear configuration space is necessarily gauge dependent and therefore unphysical. On the other hand, there is an objective way to relate the origin of the MAB phase effect locally in nuclear configuration space using the open path Berry phase $\gamma_{n}$ for the corresponding electronic Born-Oppenheimer state vector $|\widetilde{n}(\theta)\rangle=e^{i \xi(\theta)}|n(\theta)\rangle$. Here, we assume $\xi(\theta)$ to be differentiable along the path but otherwise arbitrary. The noncyclic Berry phase is defined by removing the accumulation of local phase changes from the total phase and is testable in polarimetry [31, 35] or in interferometry [36, 37]. We obtain for 
$|\widetilde{n}(\theta)\rangle$

$$
\gamma_{n}=\arg \left\langle\widetilde{n}\left(\theta_{0}\right) \mid \widetilde{n}(\theta)\right\rangle+i \int_{\theta_{0}}^{\theta}\left\langle\widetilde{n}\left(\theta^{\prime}\right)\left|\frac{\partial}{\partial \theta^{\prime}}\right| \widetilde{n}\left(\theta^{\prime}\right)\right\rangle d \theta^{\prime}=\arg \left\langle n\left(\theta_{0}\right) \mid n(\theta)\right\rangle,
$$

by using Eq. (3). Clearly, $\gamma_{n}$ is locally gauge invariant as it is independent of $\xi(\theta)$. It corresponds to phase jumps of $\pi$ at points across which the realvalued quantity $\left\langle n\left(\theta_{0}\right) \mid n(\theta)\right\rangle$ goes through zero and changes sign. In the case where an even number of $\pi$ phase jumps occurs, the nuclear factor $\chi(\theta)$ is single-valued and there is no MAB effect. On the other hand, for an odd number of such jumps, there is a physically nontrivial sign change for such a loop. Thus, the presence of a nontrivial MAB effect could be explained locally as it requires the existence of points along the nuclear path where the electronic states at $\theta_{0}$ and $\theta$ become orthogonal. This assignment of electronic Berry phase shift is gauge invariant at each point along the nuclear path and thus experimentally testable in principle. It shows that MAB does not obey the criterion (T2) for a topological phase effect.

\section{$3 \quad$ Preferred gauge}

The assignment of a gauge invariant electronic Berry phase for any open portion of the closed nuclear path suggests that MAB is not topological and that it might be meaningful to introduce a notion of preferred gauge in this context. The corresponding preferred vector potential is defined as that whose line integral gives the open path Berry phase. This idea can be put forward in terms of a so-called gauge invariant reference section [25, 26] as follows.

First, in general terms, let $|\psi(s)\rangle$ be a normalized Hilbert space representative of the pure quantal state $\psi(s)$ tracing out the path $\mathcal{C}: s \in\left[s_{0}, s_{1}\right] \rightarrow$ $\psi(s)$ in projective Hilbert space $\mathcal{P}$. Now, if $0 \neq\left|\left\langle\psi\left(s_{0}\right) \mid \psi\left(s_{1}\right)\right\rangle\right| \leq 1$, then the Berry phase $\gamma[\mathcal{C}]$ associated with $\mathcal{C}$ is 28

$$
\begin{aligned}
\gamma[\mathcal{C}] & =\arg \left\langle\psi\left(s_{0}\right) \mid \psi\left(s_{1}\right)\right\rangle-\int_{s_{0}}^{s_{1}} \arg \langle\psi(s) \mid \psi(s+d s)\rangle \\
& =\arg \left\langle\psi\left(s_{0}\right) \mid \psi\left(s_{1}\right)\right\rangle+i \int_{s_{0}}^{s_{1}}\langle\psi(s) \mid \dot{\psi}(s)\rangle d s,
\end{aligned}
$$

where the first and second term on the right-hand side contain the global phase and the accumulation of local phase changes, respectively. This open 
path Berry phase is real-valued and reparametrization invariant 28]. It is gauge invariant and thereby measurable [31, 36, 37, 35. in that it is independent of choice of Hilbert space representative. $\gamma[\mathcal{C}]$ reduces to the cyclic Berry phase [5] in the particular case where $\left|\left\langle\psi\left(s_{0}\right) \mid \psi\left(s_{1}\right)\right\rangle\right|=1$.

A gauge invariant reference section $|\phi(s)\rangle$ is a nonlinear functional of $|\psi(s)\rangle$ defined as [25, 26]

$$
\left|\phi\left(s ; s_{0}\right)\right\rangle=\exp \left(-i \arg \left\langle\psi\left(s_{0}\right) \mid \psi(s)\right\rangle\right)|\psi(s)\rangle,
$$

which has the image $\mathcal{C}$ in $\mathcal{P} .\left|\phi\left(s ; s_{0}\right)\right\rangle$ is in one to one correspondence with $\mathcal{C}$ in that it is gauge invariant under phase transformations of $|\psi(s)\rangle$. Furthermore, by inserting Eq. (6) into Eq. (5), we obtain

$$
\gamma[\mathcal{C}]=\int_{s_{0}}^{s_{1}} \mathcal{A}\left(s ; s_{0}\right) d s
$$

where the gauge function reads

$$
\mathcal{A}\left(s ; s_{0}\right)=i\left\langle\phi\left(s ; s_{0}\right) \mid \partial_{s} \phi\left(s ; s_{0}\right)\right\rangle .
$$

Now, the one to one correspondence between the path $\mathcal{C}$ and $\left\{\left|\phi_{n}\left(s ; s_{0}\right)\right\rangle \mid\right.$ $\left.s \in\left[s_{0}, s_{1}\right]\right\}$, and the fact that the open path Berry phase can be directly expressed in terms of the vector potential $\mathcal{A}\left(s ; s_{0}\right)$, suggest that the gauge invariant reference section defined in Eq. (6) has a special status: it is natural to regard $\left|\phi_{n}\left(s ; s_{0}\right)\right\rangle$ and $\mathcal{A}\left(s ; s_{0}\right)$ to constitute a 'preferred gauge' for the measurable open path Berry phase. In doing so, one should take notice that the functional form of the preferred vector potential $\mathcal{A}\left(s ; s_{0}\right)$ depends in general upon $\psi\left(s_{0}\right)$, but is otherwise unique up to an additional term whose integral over the interval $\left[s_{0}, s_{1}\right]$ equals an integral multiple of $2 \pi$. In particular, once $\psi\left(s_{0}\right)$ has been chosen the corresponding phase factor is gauge invariant under phase transformations of $|\psi(s)\rangle$.

We next apply this idea and calculate the gauge invariant electronic reference section relevant for $\mathrm{MAB}$ and its concomitant vector potential in the case of real electronic Hamiltonians. Let the pseudorotational angle $\theta \in\left[\theta_{0}, \theta_{0}+2 \pi\right]$ parametrize a closed path $\mathcal{C}_{N}$ in nuclear configuration space. Let $|n(\theta)\rangle=R(\theta)|n\rangle$ be a Hilbert space representative of a nondegenerate electronic energy eigenstate along this path. Under these assumptions, consider a finite portion $\Delta \mathcal{C}_{N}$ of $\mathcal{C}_{N}$. First, suppose that $\left\langle n\left(\theta_{0}\right) \mid n(\theta)\right\rangle \neq 0, \theta \in \Delta \mathcal{C}_{N}$. Along such a $\Delta \mathcal{C}_{N}$, the gauge invariant reference section reads

$$
\left|\phi_{n}\left(\theta ; \theta_{0}\right)\right\rangle=\exp \left(-i \arg \left\langle n\left(\theta_{0}\right) \mid n(\theta)\right\rangle\right)|n(\theta)\rangle= \pm|n(\theta)\rangle,
$$


where the sign is independent of $\theta$. This follows since $\left\langle n\left(\theta_{0}\right) \mid n(\theta)\right\rangle$ is realvalued and may only change sign where it vanishes. Inserting Eq. (92) into Eq. (8), we obtain $\mathcal{A}_{n}\left(\theta ; \theta_{0}\right)=0, \theta \in \Delta \mathcal{C}_{N}$.

Now, suppose there is a single isolated point $\theta_{k} \in \Delta \mathcal{C}_{N}$ across which $\left\langle n\left(\theta_{0}\right) \mid n\left(\theta_{k}\right)\right\rangle$ goes through zero and changes sign. For such a point, it follows that

$$
\left|\phi_{n}\left(\theta ; \theta_{0}\right)\right\rangle= \pm \exp \left(i \pi h_{s}\left(\theta-\theta_{k}\right)\right)|n(\theta)\rangle, \theta \in \Delta \mathcal{C}_{N},
$$

where $h_{s}$ is the unit step function. This yields

$$
\mathcal{A}_{n}\left(\theta ; \theta_{0}\right)=-\pi \delta\left(\theta-\theta_{k}\right), \theta \in \Delta \mathcal{C}_{N}
$$

where we have used that $\partial_{\theta} h_{s}\left(\theta-\theta_{k}\right)=\delta\left(\theta-\theta_{k}\right)$.

Extending this to the whole closed path $\mathcal{C}_{N}$, we may assume it contains $K$ angles $\theta_{1}, \ldots, \theta_{K}$, all across which $\left\langle n\left(\theta_{0}\right) \mid n(\theta)\right\rangle$ goes through zero and changes sign. Then, the gauge invariant reference section reads

$$
\left|\phi_{n}\left(\theta ; \theta_{0}\right)\right\rangle=\exp \left(i \pi \sum_{k=1}^{K} h_{s}\left(\theta-\theta_{k}\right)\right)|n(\theta)\rangle, \theta \in \mathcal{C}_{N}
$$

with the corresponding preferred vector potential

$$
\mathcal{A}_{n}\left(\theta ; \theta_{0}\right)=-\pi \sum_{k=1}^{K} \delta\left(\theta-\theta_{k}\right), \theta \in \mathcal{C}_{N}
$$

Notice that $\mathcal{A}_{n}\left(\theta ; \theta_{0}\right)$ is a local quantity as it only depends upon the angles $\theta_{1}, \ldots, \theta_{K}$, whose location is gauge invariant once the 'initial' electronic eigenstate $n\left(\theta_{0}\right)$ has been chosen.

There is a nontrivial MAB effect if and only if $K$ is odd, since

$$
\gamma_{n}\left[\mathcal{C}_{N}\right]=\oint_{\mathcal{C}_{N}} \mathcal{A}_{n} d \theta=-K \pi
$$

It is important to notice that this criterion for a nontrivial MAB is only fulfilled if the effective nuclear motion has physical access to the whole closed path $\mathcal{C}_{N}$. To see this, let $\left\langle n\left(\theta_{0}\right) \mid n(\theta)\right\rangle$ change sign at the angles $\theta_{1}, \ldots, \theta_{K}$ along $\mathcal{C}_{N}$. In the preferred gauge the effective Hamiltonian operator for the nuclear motion in the $\theta$ direction reads

$$
H_{n}=\left\langle\phi_{n}\left(\theta ; \theta_{0}\right)|H| \phi_{n}\left(\theta ; \theta_{0}\right)\right\rangle=\frac{1}{2}\left[p_{\theta}+\mathcal{A}_{n}\left(\theta ; \theta_{0}\right)\right]^{2}+E_{n}(\theta) .
$$


Suppose $E_{n}(\theta)$ comprises an infinite potential barrier over the angular range $\theta \in[\vartheta, \vartheta+\Delta \vartheta], 0<\Delta \vartheta<2 \pi$, creating an inaccessible part $\mathcal{C}_{N}(\Delta \vartheta)$ of $\mathcal{C}_{N}$ for the nuclear motion. Then, it is consistent with the boundary condition $\chi(\vartheta)=\chi(\vartheta+\Delta \vartheta)=0$ to absorb the vector potential $\mathcal{A}_{n}$ into the phase of the nuclear factor.

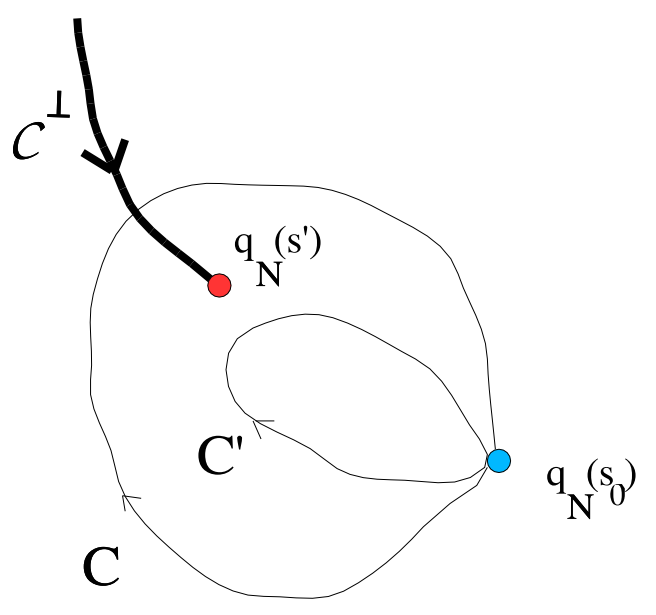

Figure 1: Single line $\mathcal{C}^{\perp}: s \in[0,1] \rightarrow q_{N}(s)$ in nuclear configuration space for which $\mathcal{A}\left(q_{N}(s) ; q_{N}\left(s_{0}\right)\right)=-\pi \delta\left(q_{N}-q_{N}(s)\right)$, for $0 \leq s<s^{\prime}<1$, and $\mathcal{A}\left(q_{N}(s) ; q_{N}\left(s_{0}\right)\right)=0$, for $s^{\prime}<s \leq 1$. For any closed path $\mathrm{C}$ that cross $\mathcal{C}^{\perp}$, the electronic eigenvector picks up a sign implying a degeneracy on any surface that has $\mathrm{C}$ as boundary. For any closed path $\mathrm{C}^{\prime}$ that does not cross $\mathcal{C}^{\perp}$, no such sign change occur. From Longuet-Higgins theorem [4] follows that $q_{N}\left(s^{\prime}\right)$ is a point of electronic degeneracy.

Now, what is the origin of a nontrivial gauge invariant reference section? In part this can be answered in terms of the following relationship between the gauge invariant reference section and degeneracy points where two or more electronic potential energy surfaces cross (for an analysis of the related connection between degeneracies and singularities of the electronic eigenvectors, see [38]). Let $q_{N}$ be the internal nuclear coordinates and consider a line $\mathcal{C}^{\perp}: s \in[0,1] \rightarrow q_{N}(s)$ in nuclear configuration space for which $\mathcal{A}\left(q_{N}(s) ; q_{N}\left(s_{0}\right)\right)=-\pi \delta\left(q_{N}-q_{N}(s)\right)$, for $0 \leq s<s^{\prime}<1$, and $\mathcal{A}\left(q_{N}(s) ; q_{N}\left(s_{0}\right)\right)=0$, for $s^{\prime}<s \leq 1$, see Fig. 1. Now, any path $\mathrm{C}$ in the 
Born-Oppenheimer regime that starts and ends at the reference point $q_{N}\left(s_{0}\right)$ and cross $\mathcal{C}^{\perp}$ once, must be associated with a sign change of the electronic eigenvector and thus must enclose at least one point of degeneracy on any surface that has $\mathrm{C}$ as boundary, as implied by the Longuet-Higgins theorem 4]. On the other hand, there is no such sign change originating from $\mathcal{C}^{\perp}$ for a closed path $\mathrm{C}^{\prime}$ that does not cross $\mathcal{C}^{\perp}$, which implies that there must be one less degeneracy enclosed by such a path than by C. Thus, $q_{N}\left(s^{\prime}\right)$ is a degeneracy point.

To illustrate the gauge invariant reference section for MAB, let us revisit the linear + quadratic $E \otimes \varepsilon$ Jahn-Teller effect, which is known to exhibit a nontrivial MAB structure. There, the symmetry induced degeneracy of two electronic states $(E)$ is lifted by their interaction with a doubly degenerate vibrational mode $(\varepsilon)$. In the vicinity of the degeneracy point at the symmetric nuclear configuration, this may be modeled by the vibronic Hamiltonian [39]

$$
H=\frac{1}{2} p_{r}^{2}+\frac{1}{2 r^{2}} p_{\theta}^{2}+\frac{1}{2} r^{2}+\Delta \mathcal{E}(r, \theta)\left(\cos \alpha(r, \theta) \sigma_{x}+\sin \alpha(r, \theta) \sigma_{z}\right) .
$$

Here, $(r, \theta)$ are polar coordinates of the vibrational mode, $\left(p_{r}, p_{\theta}\right)$ the corresponding canonical momenta, $k \geq 0$ and $g \geq 0$ are the linear and quadratic vibronic coupling strength, respectively. $\Delta \mathcal{E}$ and $\alpha$ are given by

$$
\begin{aligned}
\Delta \mathcal{E}(r, \theta) & =\sqrt{k^{2} r^{2}+k g r^{3} \cos 3 \theta+\frac{1}{4} g^{2} r^{4}} \\
\Delta \mathcal{E}(r, \theta) e^{i \alpha(r, \theta)} & =k r \cos \theta+\frac{1}{2} g r^{2} \cos 2 \theta+i\left(k r \sin \theta-\frac{1}{2} g r^{2} \sin 2 \theta\right) .(17
\end{aligned}
$$

The electronic degrees of freedom are described by Pauli operators defined in terms of the diabatic electronic states $|0\rangle$ and $|1\rangle$ as $\sigma_{x}=|0\rangle\langle 1|+| 1\rangle\langle 0|$, $\sigma_{y}=-i|0\rangle\langle 1|+i| 1\rangle\langle 0|$, and $\sigma_{z}=|0\rangle\langle 0|-| 1\rangle\langle 1|$. Diagonalizing the electronic part of $H$ yields the electronic eigenvectors

$$
\begin{aligned}
|+(\alpha)\rangle & =\cos \frac{\alpha}{2}|0\rangle+\sin \frac{\alpha}{2}|1\rangle, \\
|-(\alpha)\rangle & =-\sin \frac{\alpha}{2}|0\rangle+\cos \frac{\alpha}{2}|1\rangle
\end{aligned}
$$

with corresponding energies $E_{ \pm}=\frac{1}{2} r^{2} \pm \Delta \mathcal{E}(r, \theta)$ and where we have put $\alpha \equiv \alpha(r, \theta)$ for brevity. The phase $\alpha$ is undefined only if $\Delta \mathcal{E}(r, \theta)=0$, corresponding to the degeneracy points at $r=0$ and $r=2 \mathrm{k} / \mathrm{g}$ for $\theta=$ $\pi / 3, \pi, 5 \pi / 3$. 


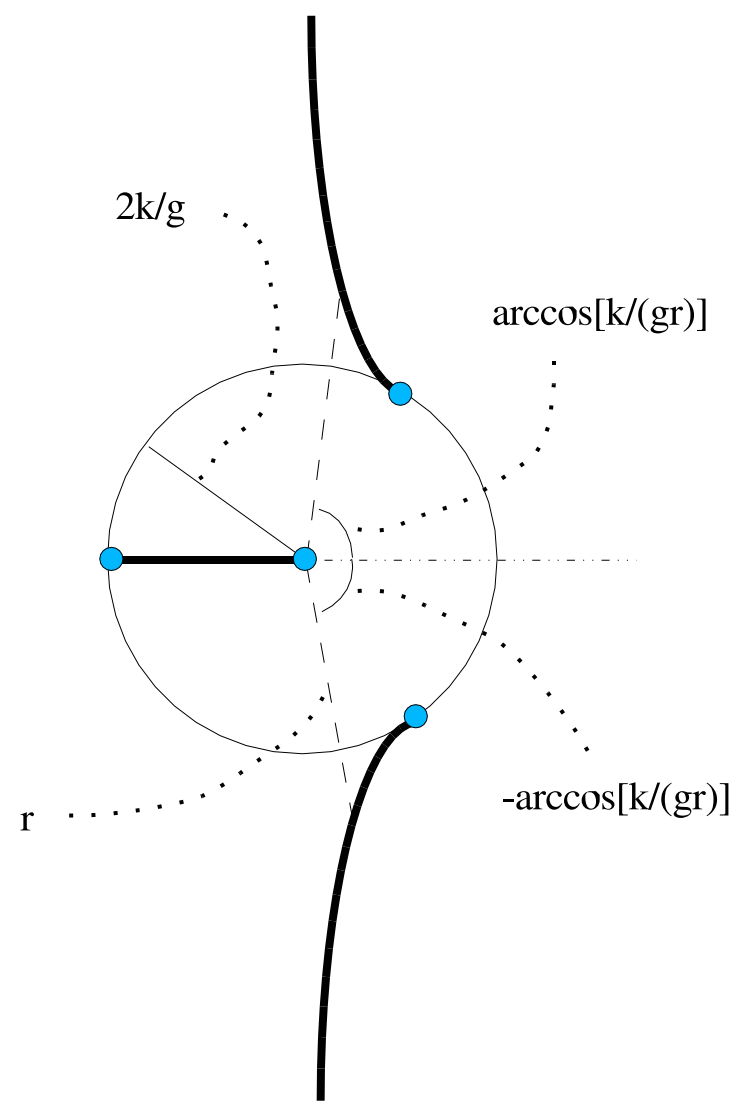

Figure 2: Lines of sign change and degeneracy points in the linear + quadratic $E \otimes \varepsilon$ Jahn-Teller model for any of the two Born-Oppenheimer states. Degeneracies are indicated by small circles; they occur at the origin and at $r=2 \mathrm{k} / \mathrm{g}$ for angles $\theta=\pi / 3, \pi, 5 \pi / 3$. The electronic reference state is chosen at an arbitrary point along the dashed-dotted line $(\theta=0)$. The thick continuous lines correspond to points where $\left\langle \pm\left(\alpha_{0}\right) \mid \pm(\alpha)\right\rangle$ goes through zero and changes sign. These lines of sign change end either at infinity or at a degeneracy. For a circular closed path with $r>2 k / g$, there is an even number of jumps for this path, and consequently no MAB effect for the corresponding nuclear motion along the path. On the other hand, for any circular closed path with $r<2 \mathrm{k} / \mathrm{g}$ there is a single node at $\theta=\pi$, assigning a nontrivial MAB effect. 
To calculate the gauge invariant reference section, we may choose $\theta_{0}=0$ so that $\alpha_{0} \equiv \alpha\left(r_{0}, \theta_{0}\right)=0$, independent of $r_{0}$. In this case, we obtain

$$
\left\langle \pm\left(\alpha_{0}\right) \mid \pm(\alpha)\right\rangle=\cos \frac{\alpha}{2} .
$$

This vanishes for $\alpha=\pi$ corresponding to

$$
\begin{aligned}
& k r \sin \theta-\frac{1}{2} g r^{2} \sin 2 \theta=0, \\
& k r \cos \theta+\frac{1}{2} g r^{2} \cos 2 \theta<0,
\end{aligned}
$$

which have the solutions $\theta^{\prime}=\pi$ for $r<2 k / g$ and $\theta^{\prime}= \pm \arccos [k /(g r)]$ for $r>2 k / g$. Since the solutions for $r<2 k / g$ are independent of $r$, they constitute a radial line whose end-points are the electronic degeneracies at the origin and at $\left(r, \theta^{\prime}\right)=(2 k / g, \pi)$, see Fig. 2. If $2 k / g<r \rightarrow \infty$, we obtain the limit angles $\theta^{\prime}= \pm \pi / 2$ at the infinity. On the other hand, if $r \rightarrow 2 k / g^{+}$, then the lines of sign change terminate at $\theta^{\prime}= \pm \pi / 3$, which are the remaining electronic degeneracies, see Fig. 2.

Next, let us consider the gauge invariant reference section and the corresponding vector potential. For $r<2 k / g$, we have

$$
\begin{aligned}
\left|\phi_{ \pm}\right\rangle & =\exp \left(i \pi h_{s}(\theta-\pi)\right)| \pm(\alpha)\rangle, \\
\mathcal{A}_{ \pm}(\theta ; 0) & =-\pi \delta(\theta-\pi),
\end{aligned}
$$

while for $r>2 k / g$, we have

$$
\begin{aligned}
\left|\phi_{ \pm}\right\rangle= & \exp \left(i \pi h_{s}(\theta-\arccos [k /(g r)])\right. \\
& \left.+i \pi h_{s}(\theta+\arccos [k /(g r)])\right)| \pm(\alpha)\rangle, \\
\mathcal{A}_{ \pm}(\theta ; 0)= & -\pi \delta(\theta-\arccos [k /(g r)])-\pi \delta(\theta+\arccos [k /(g r)]) .
\end{aligned}
$$

\section{Aharonov-Casher analogue}

It was demonstrated in [24] that for the linear or the quadratic $E \otimes \varepsilon$ JahnTeller system, there is a close analogy with the Aharonov-Casher (AC) phase effect 34 for an electrically neutral spin $-\frac{1}{2}$ particle encircling a line of charge. From this perspective, one may regard the presence (absence) of MAB effect 
in the linear (quadratic) case as a nontrivial (trivial) $\pi(2 \pi)$ AC phase shift. Here, we extend this idea to the linear + quadratic case.

In brief, the AC effect may occur when an electrically neutral spin- $\frac{1}{2}$ particle carrying a magnetic dipole moment $\mu$ encircles a straight line of charge. Under the condition that the spin is parallel to the charged line, the particle acquires along the closed path $\partial S$ the phase shift $(\hbar=1)$

$$
\gamma_{\mathrm{AC}}=\frac{\mu}{c^{2}} \oint_{\partial S}(\hat{\mathbf{n}} \times \mathbf{E}) \cdot d \mathbf{r}=\frac{\mu}{c^{2}} \iint_{S} \nabla \cdot \mathbf{E} d S=\frac{\mu \lambda}{c^{2} \epsilon_{0}}
$$

with $c$ the speed of light, $\hat{\mathbf{n}}$ the direction of the dipole, $\mathbf{E}$ the electric field, $d \mathbf{r}$ a line element along this path, $S$ any surface with $\partial S$ as boundary, $\lambda$ the enclosed charge density, and $\epsilon_{0}$ the electric vacuum permittivity.

To demonstrate the promised analogy between the MAB effect in the $E \otimes \varepsilon$ Jahn-Teller system and the AC effect, we may use the apparent spin analogy in terms of which we may notice that the electronic Hamiltonian in Eq. (16) describes a spin $-\frac{1}{2}$ under influence of an effective magnetic field that rotates around the $\sigma_{y}$ axis by the angle $\alpha(r, \theta)$. We expect the electronic Hamiltonian be fixed (possibly up to an unimportant $r$ and $\theta$ dependent scale factor) in an internal molecular frame that co-moves with this rotation. The essential point here is that, contrary to the usual case of a spin $-\frac{1}{2}$ in a rotating external magnetic field, the rotation angle $\alpha$ of this Jahn-Teller system depends upon the internal variables $r$ and $\theta$. This has the consequence that the vibronic Hamiltonian in the co-moving frame, which reads (omitting the inessential $\frac{1}{2} p_{r}^{2}$ and $\frac{1}{2} r^{2}$ terms)

$$
H^{\prime}=U^{\dagger} H U=\frac{1}{2 r^{2}}\left[p_{\theta}-\frac{1}{2} \partial_{\theta} \alpha(r, \theta) \sigma_{y}\right]^{2}+\Delta \mathcal{E}(r, \theta) \sigma_{z}
$$

with $U=\exp \left[-i \alpha(r, \theta) \sigma_{y} / 2\right]$ the unitary spin rotation operator, contains a nontrivial modification of the nuclear kinetic energy operator. Indeed, by using $H^{\prime}$ and the Heisenberg picture, this modification in turn affects the equations of motion for the electronic variables, which read

$$
\begin{aligned}
\dot{\sigma}_{x} & =-\partial_{\theta} \alpha(r, \theta) \dot{\theta} \sigma_{z}-2 \Delta \mathcal{E}(r, \theta) \sigma_{y}, \\
\dot{\sigma}_{y} & =2 \Delta \mathcal{E}(r, \theta) \sigma_{x} \\
\dot{\sigma}_{z} & =\partial_{\theta} \alpha(r, \theta) \dot{\theta} \sigma_{x},
\end{aligned}
$$

where $r^{2} \dot{\theta}=p_{\theta}-\frac{1}{2} \partial_{\theta} \alpha(r, \theta) \sigma_{y}$. The Born-Oppenheimer regime is characterized by the condition $\partial_{\theta} \alpha(r, \theta)|\dot{\theta}| \ll \Delta \mathcal{E}(r, \theta)$ that apparently breaks down 
when $\Delta \mathcal{E}(r, \theta)$ is very small, which happens close to the electronic degeneracies. From Eq. (25), it follows that the electronic motion describes the local torque due to an effective magnetic field $\mathbf{B}_{\text {eff }}=-\partial_{\theta} \alpha(r, \theta) \dot{\theta} \mathbf{e}_{y}+2 \Delta \mathcal{E}(r, \theta) \mathbf{e}_{z}$ seen by the electronic variables in the rotating frame. The large $z \mathrm{com}$ ponent of $\mathbf{B}_{\text {eff }}$ depends only on the energy difference $\Delta \mathcal{E}(r, \theta)$ between the two electronic states and is thus irrelevant to MAB. On the other hand, the small $y$ component corresponds exactly to the MAB effect and gives rise to a $\boldsymbol{\sigma} \cdot\left(\mathbf{v} \times \mathbf{E}_{\text {eff }}\right)$ term for the electronic variables in the co-moving frame in the $x-z$ plane. Explicitly, we have

$$
\mathbf{E}_{\mathrm{eff}}=\frac{\partial_{\theta} \alpha(r, \theta)}{2 r} \mathbf{e}_{r}+E_{\theta}(r, \theta) \mathbf{e}_{\theta}
$$

where we have left out the explicit form of the $\theta$ component of $\mathbf{E}_{\text {eff }}$, as it does not contribute to the MAB phase effect. The effect of this field is equivalent to that of an $\mathrm{AC}$ system that consists of four charged lines in the $y$ direction sitting at the four conical intersections at $r=0$ and $r=2 \mathrm{k} / \mathrm{g}$ for $\theta=\pi / 3, \pi, 5 \pi / 3$, all of which with the charge per unit length being proportional to $\frac{1}{2}$. Thus, the MAB effect for the $E \otimes \varepsilon$ Jahn-Teller system resembles exactly that of the AC effect for an electrically neutral spin $-\frac{1}{2}$ particle encircling a certain configuration of charged lines perpendicular to the plane of motion.

Note that the phase shift $\gamma_{\mathrm{AC}}$ only depends upon the enclosed charge, but is independent of the shape of the dipole's path. On the other hand, the dipole feels the gauge invariant electric field $\mathbf{E}$, which defines a preferred gauge in terms of the gauge invariant effective vector potential $\left(\mu / c^{2}\right) \hat{\mathbf{n}} \times \mathbf{E}$ and which causes a nontrivial, essentially local and nontopological autocorrelation among the spin variables, as demonstrated in [29]. From this perspective, we believe that the present analogy between the $\mathrm{AC}$ and MAB effects further strengthens the local and nontopological interpretation of MAB for this Jahn-Teller system.

\section{Conclusions}

Arguably one of the most intriguing discoveries of the second half of the $20 t h$ century was that of a nonlocal and topological interference effect for a charged particle moving around a magnetic flux line, made by Aharonov and Bohm (AB) 23]. Analogues of this remarkable effect have since then been 
found, such as the Aharonov-Casher (AC) effect [34] and its Maxwell dual, the so-called He-McKellar-Wilkens (HMW) effect [40, 41]. Common to these analogue effects are that they only occur under certain restrictions on some additional degree of freedom: the spin direction for $\mathrm{AC}$ and the direction of an electric dipole for HMW. These additional restrictions turn out to make these effects essentially different from the standard $\mathrm{AB}$, in that they do not obey all the properties for being nonlocal and topological.

Does the molecular Aharonov-Bohm (MAB) effect share the fate of these other analogue effects? This question was examined quite recently by the present author [24], in the particular case of the $E \otimes \varepsilon$ Jahn-Teller system. In identifying the restrictions for this system, which are the conditions of attaining the Born-Oppenheimer regime and the electronic state being in one of two instantaneous energy eigenstates, this issue can be addressed very much along the line of the other analogue effects. The outcome was: the $\mathrm{MAB}$ effect for this system is neither nonlocal nor topological in the sense of the standard $\mathrm{AB}$ effect. In this paper, this result has been generalized to any molecular system with real electronic Hamiltonian and where a nontrivial MAB effect shows up. In addition, a notion of preferred gauge for such systems, defined by an effective vector potential whose open path integral is the open path Berry phase for the electronic motion, has been suggested.

One of the early motivations preceding the analysis in [24] was to examine whether there is a relation between the MAB effect in the $E \otimes \varepsilon$ Jahn-Teller system and the AC effect, based upon the simple observation that the original vibronic Hamiltonian for this Jahn-Teller system resembles exactly that of an electrically neutral spin $-\frac{1}{2}$ in a certain field configuration. Indeed, by transforming to a molecular frame that co-moves with the nuclear pseudorotation, this analogue was made explicit in [24] in the case of either linear or quadratic coupling. In the present paper, this result has been extended to the linear + quadratic case, leading to an anisotropic effective electric field originating from four charged lines sitting at the four conical intersections in nuclear configuration space.

\section{Acknowledgments}

I wish to express my deep gratitude to Prof. Osvaldo Goscinski, especially for introducing me to the subject of geometric phases and how they appear in molecular systems, but also for numerous discussions and collaboration 
during the past 10 years. Osvaldo's great impact on my scientific thinking makes it a very special honour to dedicate this paper on his $65 t h$ birthday. I also wish to thank Mauritz Andersson, Henrik Carlsen, Marie Ericsson, Gonzalo García de Polavieja, Magnus Hedström, and Niklas Johansson for discussions and collaboration over the years on issues related to the present paper. This work was supported by the Swedish Research Council.

\section{References}

[1] H.C. Longuet-Higgins, U. Öpik, M.H.L. Pryce and R.A. Sack, Proc. Roy. Soc. London Ser. A 244, 1 (1958).

[2] H.C. Longuet-Higgins, Adv. Spectr. 2, 429 (1961).

[3] G. Herzberg and H.C. Longuet-Higgins, Disc. Frad. Soc. 35, 77 (1963).

[4] H.C. Longuet-Higgins, Proc. Roy. Soc. London Ser. A 344, 147 (1975).

[5] M.V. Berry, Proc. Roy. Soc. London Ser. A 392, 45 (1984).

[6] B. Kendrick, Phys. Rev. Lett. 79, 2431 (1997).

[7] H. von Busch, V. Dev, H.-A. Eckel, S. Kasahara, J. Wang, W. Demtröder, P. Sebald, and W. Meyer, Phys. Rev. Lett. 81, 4584 (1998).

[8] A. Kupperman and Y.M. Wu, Chem. Phys. Lett. 205, 577 (1993).

[9] B. Kendrick and R.T. Pack, J. Chem. Phys. 104, 7475 (1996).

[10] S. Adhikari and G.D. Billing, Chem. Phys. 259, 149 (2000).

[11] E. Sjöqvist and O. Goscinski, Chem. Phys. 186, 17 (1994).

[12] F.S. Ham, Phys. Rev. Lett. 58, 725 (1987).

[13] F.S. Ham, J. Phys.: Condens.Matter 2, 1163 (1990).

[14] P. De Los Rios, N. Manini, and E. Tosatti, Phys. Rev. B 54, 7157 (1996).

[15] C.P. Moate, M.C.M. O'Brien, J.L. Dunn, C.A. Bates, Y.M. Liu, and V.Z. Polinger, Phys. Rev. Lett. 77, 4362 (1996). 
[16] A.J. Stone, Proc. Roy. Soc. London Ser. A 351, 141 (1976).

[17] A.J.C. Varandas, J. Tennyson, and J.N. Murrell, Chem. Phys. Lett. 61, 431 (1979).

[18] S. Xantheas, S.T. Elbert, and K. Ruedenberg, J. Chem. Phys. 93, 7519 (1990).

[19] M. Ceotto and F.A. Gianturco, J. Chem. Phys. 112, 5820 (2000).

[20] N. Johansson and E. Sjöqvist, Phys. Rev. Lett. 92, 060406 (2004).

[21] C.A. Mead and D.G. Truhlar, J. Chem. Phys. 70, 2284 (1979);

[22] C.A. Mead, Chem. Phys. 49, 23 (1980); Ibid. 49, 33 (1980).

[23] Y. Aharonov and D. Bohm, Phys. Rev. 115, 485 (1959).

[24] E. Sjöqvist, Phys. Rev. Lett. 89, 210401 (2002).

[25] A.K. Pati, J. Phys. A 28, 2087 (1995).

[26] A.K. Pati, Phys. Rev. A 52, 2576 (1995).

[27] J. Samuel and R. Bhandari, Phys. Rev. Lett. 60, 2339 (1988).

[28] N. Mukunda and R. Simon, Ann. Phys. (N.Y.) 288, 205 (1993).

[29] M. Peshkin and H.J. Lipkin, Phys. Rev. Lett. 74, 2847 (1995).

[30] E. Sjöqvist and M. Hedström, Phys. Rev. A 56, 3417 (1997).

[31] G. García de Polavieja and E. Sjöqvist, Am. J. Phys. 66, 431 (1998).

[32] R. Englman and A. Yahalom, Phys. Rev. A 60, 1802 (1999);

[33] R. Englman, A. Yahalom, and M. Baer, Eur. Phys. J. D 8, 1 (2000).

[34] Y. Aharonov and A. Casher, Phys. Rev. Lett. 53, 319 (1984).

[35] P. Larsson and E. Sjöqvist, Phys. Rev. A 68, 042109 (2003).

[36] A.G. Wagh, V.C. Rakhecha, P. Fischer, and A. Ioffe, Phys. Rev. Lett. 81, 1992 (1998). 
[37] E. Sjöqvist, Phys. Lett. A 286, 4 (2001).

[38] L. Yin and O. Goscinski, Int. J. Quantum Chem. 37, 249 (1990).

[39] J.W. Zwanziger and E.R. Grant, J. Chem. Phys. 87, 2954 (1987).

[40] X.G. He and B.H.J. McKellar, Phys. Rev. A 47, 3424 (1993).

[41] M. Wilkens, Phys. Rev. Lett. 72, 5 (1994). 